\title{
Circunferência da Cintura, uma Medida Simples para a Obesidade Infantil?
}

Waist Circumference: A Simple Measure for Childhood Obesity?

\author{
Luciana Nicolau Aranha ${ }^{1 \oplus}$ e Gláucia Maria Moraes de Oliveira ${ }^{1 \oplus}$ \\ Universidade Federal do Rio de Janeiro, ${ }^{1}$ Rio de Janeiro, RJ - Brasil \\ Minieditorial referente ao artigo: Os Percentis e Pontos de Corte da Circunferência Abdominal para Obesidade em uma Ampla Amostra de \\ Estudantes de 6 a 10 Anos de Idade do Estado de São Paulo, Brasil
}

A obesidade infantil continua aumentando em todas as regiões do mundo, sendo considerada um dos grandes desafios de saúde pública. A prevalência aumentou de menos de 1\% em 1975 para 5,6\% em meninas e 7,8\% em meninos em 2016. ${ }^{1}$ No Brasil, os dados também são preocupantes, uma vez que no último levantamento oficial realizado pelo Instituto Brasileiro de Geografia e Estatística (IBGE), constatouse que entre 2008 e 2009, 51,4\% dos meninos e 43,8\% das meninas com idade entre 5 a 9 anos apresentavam sobrepeso ou obesidade. ${ }^{2}$

Crianças e adolescentes com obesidade têm cinco vezes mais chances de serem obesos quando adultos. ${ }^{3}$ Além do mais, a obesidade na infância está associada com a elevação da pressão arterial, resistência à insulina, diabetes mellitus, dislipidemia e com o aumento da morbimortalidade cardiovascular na idade adulta. ${ }^{4}$ Por isso, é importante identificar o excesso de gordura corporal nesta população e criar estratégias para prevenir o desenvolvimento de doenças crônicas no futuro.

Com o objetivo de detectar crianças e adolescentes com risco cardiometabólico, sugeriu-se o uso de indicadores antropométricos como ferramentas de triagem epidemiológica, uma vez que são métodos não invasivos, de baixo custo e de fácil aplicação. ${ }^{5,6}$ A circunferência da cintura (CC) por exemplo, é um indicador de adiposidade central relacionada a complicações metabólicas da obesidade na população pediátrica. ${ }^{7,8}$ Porém, ainda não existem pontos de corte de CC padronizados para classificação de adiposidade abdominal em crianças e adolescentes, o que torna o seu uso limitado.

Estudos descrevendo valores de percentis para CC têm apresentado resultados diferentes, uma vez que os valores de CC podem ser influenciados por idade, sexo e grupos étnicos, ${ }^{9-11}$ dificultando o estabelecimento de valores de referência globais para essa medida antropométrica.

Na edição atual dos Arquivos Brasileiros de Cardiologia, Santos et al., ${ }^{12}$ publicaram estudo longitudinal, realizado com 22.000 crianças (11.199 meninos) com idades entre

\section{Palavras-chave}

Criança; Adolescente; Obesidade Infantil; Sobrepeso; Fatores de Risco; Pesos e Medidas Corporais; Pressão Arterial.

Correspondência: Gláucia Maria Moraes de Oliveira • Universidade Federal do Rio de Janeiro - R. Prof. Rodolpho P. Rocco, 255 $8^{\circ}$. Andar - Sala 6, UFRJ. CEP 21941-913, Cidade Universitária, RJ - Brasil E-mail: glauciam@cardiol.br, glauciamoraesoliveira@gmail.com

DOI: https://doi.org/10.36660/abc.20200031
6 e 10 anos de idades, matriculadas em escolas públicas e particulares de 13 cidades do estado de São Paulo. Os autores apresentaram curvas de referência da CC específicas para idade e sexo e pontos de corte para identificar crianças com risco de obesidade. Os autores descreveram que aproximadamente $30 \%$ das crianças apresentaram excesso de gordura, sendo classificados com sobrepeso ou obesidade, conforme o índice de massa corporal. As análises da curva ROC mostraram o percentil 75 como ponto de corte ideal para risco de sobrepeso e obesidade e que a obesidade é claramente diagnosticada nas crianças com a CC classificada a partir do percentil $85 .^{12}$

Ao comparar as curvas da CC (percentil 50) com os resultados de um estudo brasileiro realizado com 2919 escolares com idade entre 7 a 10 anos em $2007,{ }^{13}$ na cidade de Florianópolis, Santos et al., ${ }^{12}$ observaram que as curvas atuais de percentis foram mais elevadas, chegando a um aumento de 4,0 cm nas meninas aos 10 anos de idade. Diferenças metodológicas podem explicar estes valores discrepantes, embora os autores de ambos os estudos utilizaram a mesma metodologia para a aferição da CC. No entanto, a população brasileira tem um alto grau de miscigenação e, como citado acima, os valores de CC podem ser influenciados pela etnia, o que poderia explicar a diferença desses resultados.

Como mencionado, poucos estudos no Brasil descreveram valores de ponto de corte da CC em uma grande amostra com vários grupos etários, o que justifica a importância dessas investigações para a literatura científica. Porém, como os próprios autores relataram como limitação, os valores das curvas de percentis foram determinados com base em uma amostra de crianças do estado de São Paulo, sendo aconselhável para a generalização dos resultados, utilizar amostras representativas de todos os estados do Brasil. Além disso, os valores propostos precisarão ser validados em outra população com características semelhantes.

Recentemente, $\mathrm{Xi}$ et al., ${ }^{14}$ propuseram pontos de cortes internacionais de $\mathrm{CC}$ específicos por idade e sexo para definir obesidade central, com base em dados de 113.453 crianças e adolescentes, com idades entre 4 e 20 anos, de oito países de diferentes regiões: Bulgária, China, Irã, Coréia, Malásia, Polônia, Seychelles e Suíça. Os autores estabeleceram o percentil 90 como ponto de corte para identificar a obesidade central nessa população, e verificaram que esse percentil em crianças com peso normal teve um bom desempenho para prever o risco cardiovascular, sugerindo que possa ser útil para avaliar a adiposidade abdominal em crianças e adolescentes em diferentes países. 


\section{Referências}

1. NCD Risk Factor Collaboration (NCD-RisC). Worldwide trends in body-mass index, underweight, overweight, and obesity from 1975 to 2016: a pooled analyses of 2416 population-based measurement studies in 128.9 million children, adolescentes, and adults. Lancet. 2017; 390(10113):2627-42.

2. Instituto Brasileiro de Geografia e Estatística (IBGE). Pesquisa de Orçamentos Familiares 2008-2009: antropometria e estado nutricional de crianças, adolescentes e adultos no Brasil. Rio de Janeiro, 2010.

3. Simmonds M, Llevellyn A, Owen CG, Woolacott N. Predcting adult obesity from childhood obesity: a systematic review and meta-analysis. Obes Rev. 2016; 17 (2): 95-107.

4. Sommer A, Twig G. The impact of childhood and adolescent obesity on cardiovascular risk in adulthood: a systematic review. Curr Diab Rep. 2018; $18(10): 91$.

5. Lichtenauer M, Wheatley SD, Martyn-St James M, Duncan MJ, Cobayashi F, Berg G, et al. Efficacy of anthrometric measures for identifying cardiovascular disease risk in adolescentes: review and meta-analysis. Minerva Pediatr. 2018; 70(4):371-82

6. De Quadros TMB, Gordia AP, Andaki ACR, Mendes EL, Mota J, Silva LR. Utility of anthropometric indicators to screen for clustered cardiometabolic risk factors in children and adolescentes. J Pediatr Endocrinol Metab. 2019;32(1):49-55.

7. Burgos MS, Burgos LT, Camargo MD, Franke SI, Prá D, Silva AM, et al. Relationship between anthropometric measures and cardiovascular risk factors in children and adolescentes. Arq Bras Cardiol. 2013;101(4):288-96.
8. Sardinha LB, Santos DA, Silva AM, Grøntved A, Andersen LB, Ekelund U. A comparasion between BMI, Waist Circumference, and Waist-to-Height Ratio for identifying cardio-metabolic risk in children and adolescentes. PLos One.2016; 11(2):e0149351

9. Fernández JR, Redden DP, Pietrobelli A, Allison DB. Waist circumference percentiles in nationally representative samples of African-American, European-American, and Mexican children and adolescentes. J Pediatr. 2004;145(4):439-44

10. Nawarcycz T, Haas GM, Krzyzzniak A, Schwandt P, Ostrowska-Nawarycz L. Waist circumference and waist-to-height ratio distribuitions in Polish and German schoolchildren: comparative analysis. Int J Prev Med.2013:4(7):786-96.

11. Ramírez-Vélez, Moreno-Jiménez J, Correa-Bautista JE, Martínez-Torres J, González-Ruiz K, González-Jiménez E, et al. Using LMS tables to determine waist circumference and waist-to-height ratios in Colombian children and adolsescents: the FUPRECOL study. BMC Pediatr.2017;17(1):162.

12. Santos JLF, Valério VP, Fernandes RN, Duarte L, Assumpção AC, Guerreiro J, et al. Os percentis e pontos de corte da circunferência da cintura para obesidade em uma ampla amostra de estudantes de 6 a 10 anos de idade do estado de São Paulo, Brasil. Arq Bras Cardiol. 2020; 114(3):530-537

13. de Assis MA, Rolland-Cachera MF, de Vaconcellos FA, Bellisle F, Conde W, Calvo MC, et al. Central adiposity in Brazilian schoolchildren aged 7-10 years. Br J Nutr. 2007;97(4):799-805

14. Xii B, Zong X, Kelishad R, Litwin M, Hong YM, Poh BK, etal. International waist circumference percentile cut-offs for central obesity in children and adolescents aged 6-18 years. J Clin Endocrinol Metab. 2019 Nov 14; pii: dgz195. 